\title{
Event-triggered control for a linear continuous-time system under resource constraint environment
}

\author{
Poonam Sahu \\ Electrical Engineering \\ Indian Institute of Technology, Jodhpur \\ Email: sahu.6@iitj.ac.in
}

\author{
Deepak Fulwani \\ Electrical Engineering \\ Indian Institute of Technology, Jodhpur \\ Email: df@iitj.ac.in
}

\begin{abstract}
This paper proposes a network-based eventtriggered control for the linear system. The primary feature of the proposed event-triggered rule is to broadcast the current measurements when a pre-defined condition on input error is satisfied, which can avoid the redundant control updates from the controller to the actuator. Secondly, it provides an augmented system performance by appropriate selection of design parameters. Moreover, a continuous-time dynamic event-triggered mechanism is suggested to further improve the trade-off between resource utilization and system performance. Furthermore, the finite positive time interval between two consecutive events and the stability of the system is established. A numerical example is considered to show the effectiveness of the proposed work.
\end{abstract}

Index Terms-Networked control systems (NCSs), Eventtriggered control (ETC), Event-triggered mechanism (ETM), Dynamic event-triggered mechanism (DETM).

\section{Introduction}

Event-triggered control (ETC) is one of the most significant methodologies to reduce the computation and communication resources, especially in networked control systems (NCSs). Different to the traditional time-triggered controller, where system information is transmitted continuously or periodically, in ETC, update of control law and transmission of data are driven by a pre-specified triggering algorithm. The key idea behind the triggering algorithm is to schedule the control task and broadcasts the system measurements when the evolution of error involving system information (mostly state measurements) exceeds some predefined threshold. Moreover, the fundamental concept of ETC was developed in [1], and the references therein.

In recent years, various triggering rules such as [1]-[6] have been investigated in literature due to its wide application in resource conservation, traffic control, packet dropouts etc. For instance, in [1], ETC updates the control law only when a certain decrease condition on Lyapunov function is guaranteed. In [2], ETC is proposed that updates the control law when a norm of state error satisfies a given mixed threshold while ensuring the asymptotic convergence of the system. In [4], [6], current measurements are transmitted and the control task is executed when a norm of estimated and measured output exceeds a well-designed threshold.

The abovementioned and most of the existing ETMs in the literature are categorized as a static event-triggered paradigm where the threshold parameter is fixed. Although the static ETC has been widely investigated in the literature, it may still lead to redundant transmission. Hence, to improve resource utilization and to further economize the network bandwidth, the threshold can be adaptively adjusted to fulfill the communication requirements while maintaining an expected performance. Some relevant works can be found in [7]-[10] where an auxiliary time-varying variable is introduced in the triggered condition. This auxiliary variable is typically considered a kind of filtered triggering signal and can enlarge the minimum inter-event time to an extent. Various adaptive ETMs are proposed in [11]-[13], whose main aim is to change the threshold adaptively as per the desired requirements.

It is noteworthy that most of the existing ETCs are based on state error, and the scheduling of the control task is decided as per the state-dependent ETMs. Since control law $u(t)=f(x, G)$, where $G$ is a vector of parameters, has states and a scaling factor contributed by some gain matrix $G$. In linear case, the controller becomes $u(t)=G x(t)$. It may happen that change in the state does not always yield a significant change in the input; thus, the update of control law can be skipped. Similarly, despite the considerable change in the inputs, control law cannot be updated until the corresponding state-based triggered condition is satisfied. Thus, with state error, ETM either causes the redundant update of the control task or prolongs the update of the control task and thereby, degrading the control performance. Intuitively, if an input error is considered in the triggered condition, system performance can be improved by avoiding redundant updates of the control law. Hence, ideally, the event-triggered condition should be based on input as the central idea is to update control law based on need. Considering this fact, we propose a new input-based ETM. A few attempts have been made in the literature [14]-[16], where input error is considered in the triggered condition. In [14], [16], a comparison of the effectiveness between the inputbased ETM and the state-based ETM is provided, where the control performance is quantified in terms of the number of 
events and cost function. Different from the existing ETMs [14]-[16], in this paper, an auxiliary variable is introduced in the triggered condition, which can adjust the threshold dynamically as per the error dynamics.

In many practical applications, while scheduling control over the networks requires longer inter-event times during heavy traffic on the network. Some outstanding dynamic state-based ETCs [7], [10], [17] are reported in the literature to control the inter-event time by adjusting an auxiliary variable as per the change in system dynamics. However, control law also has a significant impact on inter-event time. Hence, considering this, in this paper, we propose an inputbased dynamic ETC, which is an improved version of the existing method [7]. In the proposed triggered mechanism, some exponential terms are added into the derivative of the dynamic variable to increase the effect of these variables on system stability and to further extend the inter-event time between two consecutive events with regard to the traditional ETCs [1], [18]. In contrast to the state-based dynamic triggering mechanisms in [7]-[10], the proposed ETM can avoid the redundant updates of control law and reduce the usage of communication simultaneously, thus improves the system performance significantly. This can also be proved in the simulation by quantifying the obtained results on integral error norm.

Notation: For any vector $x \in \mathbb{R}^{n},\|x\|:=\sqrt{x^{T} x}$ represents its Euclidean norm. A function $f:[0, a) \rightarrow[0, \infty), a>0$ is said to be a class $K$ function, if $f$ is strictly increasing and $f(0)=0$. A function $f:[0, a) \rightarrow[0, \infty), a>0$ is said to be a class $K_{\infty}$ function if it belongs to class $K$, and $a=\infty$. Also $f(m)=\infty$ if $m \rightarrow \infty$.

\section{Preliminaries and system description}

Lemma 1. For any two vectors $M_{1} \in \mathbb{R}^{n}, M_{2} \in \mathbb{R}^{n}$ and a scalar $\psi>0$, it satisfies $2 M_{1} M_{2} \leq \psi M_{1}^{T} M_{1}+$ $\frac{1}{\psi} M_{2}^{T} M_{2}$.

Definition 1. [19] Consider a system of the form

$$
\dot{x}(t)=f(x, u), x \in \mathbb{R}^{n}, u \in \mathbb{R}^{m}
$$

A positive function $V(x)$ is said to be an ISS Lyapunov function if the following condition is satisfied.

$$
\dot{V}(x) \leq-\alpha_{3}(\|x\|)+\zeta(\|u\|)
$$

$\alpha_{3}$ and $\zeta$ are the class $K_{\infty}$ and class $K$-function respectively.

Definition 2. [18] The solutions of $\dot{x}(t)=f(x)$ are called ultimately bounded to a region $(\omega)$, if for each initial condition $x_{0} \in \mathbb{R}^{n}$, there exists a time $T\left(x_{0}\right) \geq 0$ such that any state trajectory of the system with initial condition $x_{0}$ satisfies $x(t) \in \omega, \forall t \geq T\left(x_{0}\right)$.

In this paper, we consider a continuous LTI system

$$
\dot{x}(t)=A x(t)+B u(t)
$$

where $x \in \mathbb{R}^{n}$ represents a state vector. $A \in \mathbb{R}^{n \times n}$ and $B \in \mathbb{R}^{n \times m}$ represent the state matrix and the input matrix

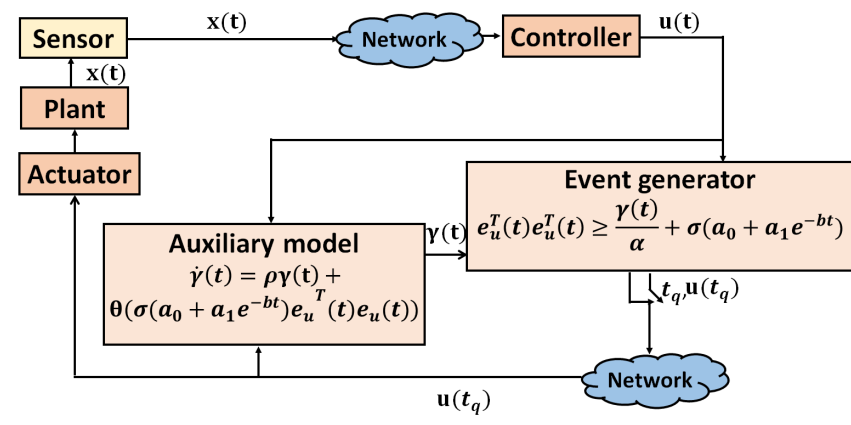

Figure 1. Complete structure of the proposed event-triggered mechanism

respectively. $u \in \mathbb{R}^{m}$ represents the input vector which is given by

$$
u(t)=G x(t)
$$

\section{Dynamic Event-triggered mechanism based on control signal}

This section proposes a new dynamic ETM where the error is input-dependent. However, for a better understanding of dynamic ETM, the counterpart static ETM is first introduced. Though static ETM has been widely investigated in the literature, very few works are reported where input error is considered in the triggered condition [15], [20]. Fig. 1 represents the complete mechanism of the proposed work. $t_{q}$ represents the sequence of triggering instants $\left\{t_{q}, t_{q+1}, \ldots\right\}$. Now introduce the error in between two consecutive triggering instants as

$$
e_{u}(t):=u\left(t_{q}\right)-u(t), t \in\left(t_{q}, t_{q+1}\right)
$$

As the control input is updated at each triggering instant; therefore, error at those triggering instants are 0 . Consider the current event is triggered at $t_{q}$ time instant, the next event will be triggered if the following condition is satisfied.

$$
t_{q+1}=\inf \left\{t: t>t_{q},\left(e_{u}^{T}(t) e_{u}(t)-\sigma\left(a_{0}+a_{1} e^{-b t}\right)>0\right\}\right.
$$

where, $a_{0}, a_{1} \geq 0, \sigma(0<\sigma<1)$ and $b$, are the static design parameters. $a_{0}$ and $a_{1}$ both can never be zero at same time. As stated in the triggering rule (6), events are generated when the evolution of error reaches the threshold value $\sigma\left(a_{0}+a_{1} e^{-b t}\right)$. Similar kind of triggering threshold is considered in [2] where error is state-dependent.

Due to the fixed design parameters $a_{0}, a_{1}$ and $\sigma$ in the triggered condition (6), the static ETM may result in a redundant transmission of the system measurements. Especially when $a_{0}$ is fixed at zero, excessive events are generated. If $a_{1}=0, a_{0} \neq 0$, then larger value of $a_{0}$ leads to system instability. Thus for static condition (6), design parameters should be carefully chosen to preserve the satisfactory performance of the system. However, it can't guarantee less number of events through out the time period. Therefore, from resource conservation perspective, it is better to design the threshold such that it can be 
dynamically adjusted as per the error dynamics as in [7], [9], [17]. In [7], [9], [17], dynamic variable depends on the system measurements and error is considered as state or output based. Different to that, in this paper we propose a dynamic ETM, where an offset variable $\gamma(t)$ is introduced whose value depends on an auxiliary model and error $\left(e_{u}^{T}(t) e_{u}(t)-\sigma\left(a_{0}+a_{1} e^{-b t}\right)\right.$. The set of event-occurrence instants for DETM is defined by

$t_{q+1}=\inf \left\{t: t>t_{q}, \theta\left(e_{u}^{T}(t) e_{u}(t)-\sigma\left(a_{0}+a_{1} e^{-b t}\right)>\gamma(t)\right\}\right.$

$\theta>0$ is a constant. Further, $\gamma(t)$ satisfies

$$
\dot{\gamma}(t)=-\rho \gamma(t)+\alpha\left[\sigma\left(a_{0}+a_{1} e^{-b t}\right)-e_{u}^{T}(t) e_{u}(t)\right]
$$

Initial condition of $\gamma(t)$ is given by $\gamma(0)>0 . \rho, \alpha$ are positive scalar.

Remark 1. Compared to the static ETM, the introduction of $\gamma(t)$ is a central idea for the proposed dynamic ETM (7) to adjust the threshold dynamically. However, for $\gamma(t)=0$, the ETM (7) becomes the static one, which can be regarded as its special case.

Remark 2. Due to an input-based error, redundant control updates are avoided. Also, the presence of absolute and asymptotic term $a_{0}$ and $a_{1}$ leads to a larger inter-event time compared to the existing rule [7], [9], [17]

Furthermore, some additional features of the dynamic variable $\gamma(t)$ are presented in the following Lemma.

Lemma 2. For any initial condition $\gamma(0)>0, \gamma(t), \forall t>0$ always remains non negative and is lower bounded by $\gamma(0) e^{-\left(\rho+\frac{\alpha}{\theta}\right) t}$.

Proof 1. Following the same procedure as mentioned in [7], [9], [17], we obtain

$$
\dot{\gamma}(t) \geq-\rho \gamma(t)-\frac{\alpha}{\theta} \gamma(t)
$$

which results

$$
\gamma(t) \geq \gamma\left(t_{q}\right) e^{-\left(\rho+\frac{\alpha}{\theta}\right)\left(t-t_{q}\right)}, t \in\left(t_{q}, t_{q+1}\right]
$$

Now using induction method we obtain

$$
\gamma(t) \geq \gamma\left(t_{q}\right) e^{-\left(\rho+\frac{\alpha}{\theta}\right)\left(t-t_{q}\right)} \geq \ldots \geq \gamma(0) e^{-\left(\rho+\frac{\alpha}{\theta}\right)(t)}
$$

As per assumption $\gamma(0)>0$. Therefore, from the above, it is cleared that $\gamma(t)>0$, this proves the Lemma.

Lemma 2 shows that the dynamic variable $\gamma(t)>0$ which leads to

$$
e_{u}^{T}(t) e_{u}(t)-\sigma\left(a_{0}+a_{1} e^{-b t}\right) \geq \frac{\gamma(t)}{\theta}>0
$$

in the dynamic triggered condition (7). Hence, it can be expected that if the current event is triggered at the same time (let $t_{q}$ time instant) in both the static and dynamic strategy, then the next event-occurrence time instant for the DETM will be no less than that generated by the SETM. This can also be easily proved by the contradiction method.

\subsection{Event-based closed-loop system}

Fig. 1 depicts the complete mechanism of the proposed work, where the controller receives the state information from the sensor and sends it to the event generator. If the event-triggered condition is satisfied, then the event is generated, and the control law is updated. The actuator receives the updated value of the control input and sends it to the plant. If the event is not triggered, then the actuator sends past updated information of the control signal.

Now define the event-triggered input $u\left(t_{q}\right)$ is written

$$
u(t):=u\left(t_{q}\right), t_{q} \leq t<t_{q+1}
$$

Using the error in (5) and event-triggered input $u\left(t_{q}\right)$, the closed-loop system is represented as

$$
\begin{aligned}
& \dot{x}(t)=A x(t)+B u\left(t_{q}\right), t \in\left[t_{q}, t_{q+1}\right) \\
& \dot{x}(t)=A_{d} x(t)+B e_{u}(t)
\end{aligned}
$$

where, $A_{d}=A+B G$.

\section{System stability}

In this section, the ultimate boundedness is achieved using Lemma 1, Lemma 2, and Definition 1.

Theorem 1. Consider the event-triggered closed loop system (14b) with controller gain $G$, and triggering rule (7). The state trajectory $x(t)$ of the system (14b) is uniformly ultimate bounded by the region with center at origin and has radius $R$ as follows.

$$
R=\Delta+\sqrt{\delta}
$$

where, $\Delta$ is an arbitrary small constant. $\delta$ is defined by

$$
\delta:=\frac{4\left(A_{d}^{T} P B\right)^{T}\left(A_{d}^{T} P B\right)}{\lambda_{\min }^{2}(Q)}
$$

Proof 2. $A_{d}$ is Hurwitz by design. For the given $Q>0$, there exists a positive definite matrix $P>0$ which is the unique solution of the following Ricatti equation

$$
A_{d}^{T} P+P A_{d}=-Q
$$

Consider an ISS Lyapunov candidate function $V(x(t), \gamma(t))$ as

$$
V(x(t), \gamma(t))=x(t)^{T} P x(t)+\gamma(t)
$$

which can be represented as

$$
\lambda_{\min }(P)\|x(t)\|^{2} \leq x(t)^{T} P x(t) \leq \lambda_{\max }(P)\|x(t)\|^{2}
$$

where $\lambda_{\min }(P)$ and $\lambda_{\max }(P)$ denote the minimum eigenvalue and maximum eigenvalue of $P$ respectively. Now differentiating $V(x(t), \gamma(t))$ we have

$$
\dot{V}(x(t), \gamma(t))=\dot{x}(t)^{T} P x(t)+x(t)^{T} P \dot{x}(t)+\dot{\gamma}(t)
$$


Using (17) and (8), $\dot{V}(x(t), \gamma(t))$ in (20) is simplified into

$$
\begin{gathered}
\dot{V}(x(t), \gamma(t))=x^{T}(t)(-Q) x(t)+2 x^{T}(t) A_{d}^{T} P B e_{u}(t) \\
-\rho \gamma(t)+\alpha\left[\left(a_{0}+a_{1} e^{-t}\right)-e_{u}(t)^{T} e_{u}(t)\right]
\end{gathered}
$$

Using Lemma 1 and (20), $\dot{V}(x(t), \gamma(t))$ is further bounded by

$$
\begin{gathered}
\dot{V}(x(t), \gamma(t)) \leq-\lambda_{\min }(Q) x^{T}(t) x(t)+\psi x^{T}(t) x(t) \\
+\frac{1}{\psi}\left(A_{d}^{T} P B\right)^{T}\left(A_{d} P B\right) e_{u}^{T}(t) e_{u}(t)-\rho \gamma(t) \\
+\alpha\left[\left(a_{0}+a_{1} e^{-t}\right)-e_{u}^{T}(t) e_{u}(t)\right]
\end{gathered}
$$

Selecting $\psi=\frac{\lambda_{\min }}{2}(Q), \dot{V}(x(t), \gamma(t))$ in (22) is reduced to

$$
\begin{aligned}
& \dot{V}(x(t), \gamma(t)) \leq-\frac{1}{2} \lambda_{\min }(Q) x(t)^{T} x(t)+\alpha\left(a_{0}+a_{1} e^{-t}\right) \\
& -\rho \gamma(t)+\left[\frac{2\left(A_{d}^{T} P B\right)^{T}\left(A_{d} P B\right)}{\lambda_{\min }(Q)}-\alpha\right] e_{u}^{T}(t) e_{u}(t)
\end{aligned}
$$

From the triggered condition (7), we have

$$
\begin{aligned}
& \dot{V}(x(t), \gamma(t)) \leq-\frac{1}{2} \lambda_{\min }(Q) x(t)^{T} x(t)+\alpha\left(a_{0}+a_{1} e^{-t}\right)- \\
& \rho \gamma(t)+\left[\frac{2\left(A_{d}^{T} P B\right)^{T}\left(A_{d} P B\right)}{\lambda_{\min }(Q)}-\alpha\right]\left[\frac{\gamma(t)}{\theta}+\left(a_{0}+a_{1} e^{-t}\right)\right] \\
& \dot{V}(x(t), \gamma(t)) \leq-\frac{1}{2} \lambda_{\min }(Q) x(t)^{T} x(t)+ \\
&+\left[\frac{2\left(A_{d}^{T} P B\right)^{T}\left(A_{d} P B\right)}{\theta \lambda_{\min }(Q)}-\frac{\alpha}{\theta}-\rho\right] \gamma(t) \\
&+\left(\frac{2\left(A_{d}^{T} P B\right)^{T}\left(A_{d} P B\right)}{\lambda_{\min }(Q)}\right)\left(a_{0}+a_{1} e^{-t}\right)
\end{aligned}
$$

Using Lemma 2, $\gamma(t)$ in (25) is substituted by

$$
\begin{aligned}
& \dot{V}(x(t), \gamma(t)) \leq-\frac{1}{2} \lambda_{\min }(Q) x(t)^{T} x(t) \\
& +\left[\frac{2\left(A_{d}^{T} P B\right)^{T}\left(A_{d} P B\right)}{\theta \lambda_{\min }(Q)}-\frac{\alpha}{\theta}-\rho\right] \gamma(0) e^{-\left(\rho+\frac{\alpha}{\theta}\right) t} \\
& +\left(\frac{2\left(A_{d}^{T} P B\right)^{T}\left(A_{d} P B\right)}{\lambda_{\min }(Q)}\right)\left(a_{0}+a_{1} e^{-t}\right)
\end{aligned}
$$

Now, we can notice from (26) that the Lyapunov derivative $\dot{V}(t)$ is strictly negative along the trajectories of the system for the region

$$
\begin{aligned}
& x(t)^{T} x(t)>\frac{2}{\lambda_{\min }(Q)}\left[\frac{2\left(A_{d}^{T} P B\right)^{T}\left(A_{d} P B\right)}{\theta \lambda_{\min }(Q)}-\frac{\alpha}{\theta}-\rho\right] \\
& \gamma(0) e^{-\left(\rho+\frac{\alpha}{\theta}\right) t}+\frac{2}{\lambda_{\min }(Q)}\left(\frac{2\left(A_{d}^{T} P B\right)^{T}\left(A_{d} P B\right)}{\lambda_{\min }(Q)}\right) \\
& \left(a_{0}+a_{1} e^{-t}\right)
\end{aligned}
$$

then the trajectories for some $k>0$, must necessarily converge into a bounded invariant set which is the ultimate region represented by the radius as

$$
\begin{aligned}
R= & \frac{2}{\lambda_{\min }(Q)}\left(\frac{2\left(A_{d}^{T} P B\right)^{T}\left(A_{d} P B\right)}{\lambda_{\min }(Q)}\right)\left(a_{0}+a_{1} e^{-t}\right) \\
& +\Delta
\end{aligned}
$$

As $t \rightarrow \infty, e^{-b t}=0$ and $e^{-\left(\rho+\frac{\alpha}{\theta}\right) t}=0$. Thus, the radius $R$ using (16) is represented by

$$
R=\Delta+(\delta)^{\frac{1}{2}}
$$

which implies state trajectories of the system are uniformly ultimate bounded by the region with radius $R$ and center at origin. Furthermore, the ultimately bounded region decreases with a decrease in the design parameter $a_{0}$. This completes the proof.

Corollary: For $a_{0}=0, a_{1} \neq 0$, state trajectories will asymptotically converge to the region around the equilibrium point when $t \rightarrow \infty$.

Theorem 2. For the event-triggered system (14b) and the triggering rule (7), the sequence of triggering instants $\left\{t_{q}, t_{q+1}, \ldots\right\}$ ensures that $\forall t>0$, the minimum interexecution time is given by

$$
\tau_{\text {min }}=\frac{1}{\Delta_{1}} \ln \left(\frac{\Delta_{1}}{\Delta_{2}}\left(a_{0}\right)^{\frac{1}{2}}+1\right)
$$

where,

$$
\Delta_{1}=\lambda_{\max }(A), \Delta_{2}=\left(\lambda_{\max }(A)+\|G\|\|B\|\right)\left\|u\left(t_{q}\right)\right\|
$$

Proof 3. We assume matrix $A \neq 0$ and $\left\|u\left(t_{q}\right)\right\| \neq 0$ for the following analysis. Consider the last event is triggered at $t=t_{q}^{t h}$ instant, it can be written as

$$
\frac{d}{d t}\left\|e_{u}(t)\right\| \leq\left\|\frac{d}{d t} e_{u}(t)\right\|, \forall t \in\left[t_{q}, t_{q+1}\right)
$$

and from (5), it follows that

$$
\frac{d}{d t}\left\|e_{u}(t)\right\| \leq\left\|\frac{d}{d t}\left(u\left(t_{q}\right)-u(t)\right)\right\|, \forall t \in\left[t_{q}, t_{q+1}\right)
$$


Since $u\left(t_{q}\right)$ remains unaltered until next event, therefore, $\frac{d}{d t} u\left(t_{q}\right)=0$ for the time interval $\left(t_{q}, t_{q+1}\right)$ and derivative of error is then bounded

$$
\begin{aligned}
\left\|\dot{e}_{u}(t)\right\| & \leq\|\dot{u}(t)\|=\|G \dot{x}(t)\|, \quad \forall t \in\left[t_{q}, t_{q+1}\right) \\
& =\left\|G\left(A x(t)+B u\left(t_{q}\right)\right)\right\| \\
& \leq\|G A x(t)\|+\left\|G B u\left(t_{q}\right)\right\|
\end{aligned}
$$$$
\left\|\dot{e}_{u}(t)\right\| \leq \lambda_{\max }(A)\|G x(t)\|+\|G\|\|B\|\left\|u\left(t_{q}\right)\right\|
$$$$
=\lambda_{\max }(A)\left\|u\left(t_{q}\right)-e_{u}(t)\right\|+\|G\|\|B\|\left\|u\left(t_{q}\right)\right\|
$$$$
\leq \lambda_{\max }(A)\left\|u\left(t_{q}\right)\right\|+\lambda_{\max }(A)\left\|e_{u}(t)\right\|+\|G\|\|B\|\left\|u\left(t_{q}\right)\right\|
$$

$$
=\left(\lambda_{\max }(A)+\|G\|\|B\|\right)\left\|u\left(t_{q}\right)\right\|+\lambda_{\max }(A)\left\|e_{u}(t)\right\|
$$

Using (31), error is further written as

$$
\left\|e_{u}(t)\right\| \leq \Delta_{1}\left\|e_{u}(t)\right\|+\Delta_{2}, t \in\left[t_{q}, t_{q+1}\right)
$$

Solving (36) with $e_{u}\left(t_{q}\right)=0$, we obtain

$$
\left\|e_{u}(t)\right\| \leq \frac{\Delta_{2}}{\Delta_{1}}\left[e^{\Delta_{1}\left(t-t_{q}\right)}-1\right], \forall t \in\left[t_{q}, t_{q+1}\right)
$$

From the ETM, the next event at $t=t_{q+1}^{t h}$ instant will occur if it satisfies

$$
e_{u}^{T}(t) e_{u}(t)=\left\|e_{u}(t)\right\|^{2} \geq \frac{1}{\theta} \gamma\left(t_{q+1}\right)+\left(a_{0}+a_{1} e^{-t_{q+1}}\right)
$$

As mentioned in Lemma 2, $\gamma(t)>0$ and it also follows that for $t \rightarrow \infty, \gamma(t) \rightarrow 0$. Thus, we can write (38) using Lemma 2 as

$$
\left\|e_{u}(t)\right\|^{2} \geq \frac{\gamma(0)}{\theta} e^{-\left(\rho+\frac{\alpha}{\theta}\right) t_{q+1}}+\left(a_{0}+a_{1} e^{-t_{q+1}}\right)
$$

Combining (39) with (37), we have

$$
\begin{aligned}
& \frac{\gamma(0)}{\theta} e^{-\left(\rho+\frac{\alpha}{\theta}\right) t_{q+1}}+\left(a_{0}+a_{1} e^{-t_{q+1}}\right) \leq\left\|e_{u}(t)\right\|^{2} \\
& \leq\left(\frac{\Delta_{2}}{\Delta_{1}}\left[e^{\Delta_{1}\left(t_{q+1}-t_{q}\right)}-1\right]\right)^{2}
\end{aligned}
$$

Now, the inter-event time is obtained as

$$
\begin{aligned}
& t_{q+1}-t_{q} \geq \frac{1}{\Delta_{1}} \\
& \ln \left[\frac{\Delta_{1}}{\Delta_{2}}\left(\frac{\gamma(0)}{\theta} e^{-\left(\rho+\frac{\alpha}{\theta}\right) t_{q+1}}+\left(a_{0}+a_{1} e^{-t_{q+1}}\right)\right)^{\frac{1}{2}}+1\right]
\end{aligned}
$$

$t_{q+1}-t_{q}$ is a finite positive scalar, thus Zeno effect is excluded. For large value of $t, \tau=t_{q+1}-t_{q}$ is bounded by

$$
t_{q+1}-t_{q} \geq \frac{1}{\Delta_{1}} \ln \left(\frac{\Delta_{1}}{\Delta_{2}}\left(a_{0}\right)^{\frac{1}{2}}+1\right)
$$

Thus, the minimum inter-execution time is given by $\tau_{\text {min }}=\frac{1}{\Delta_{1}} \ln \left(\frac{\Delta_{1}}{\Delta_{2}}\left(a_{0}\right)^{\frac{1}{2}}+1\right)$.
This completes the proof.

Proposition 1. Consider the case when $\|A\|=0$, then $\left\|e_{u}(t)\right\|$ is obtained as

$$
\left\|e_{u}(t)\right\| \leq\|G\|\|B\|\left(t-t_{q}\right)
$$

which further satisfies

$$
\frac{\gamma(0)}{\theta} e^{-\left(\rho+\frac{\alpha}{\theta}\right) t}+\left(a_{0}+a_{1} e^{-t}\right) \leq(\|G\|\|B\|)^{2}\left(t-t_{q}\right)^{2}
$$

Consider the next event is triggered at $t=t_{q+1}^{t h}$ time instant, inter-event time $t_{q+1}-t_{q}$ can be obtained from (47) as

$$
t_{q+1}-t_{q} \geq \ln \left[\frac{\bar{\zeta}}{\|G\|\|B\|}\right]^{\frac{1}{2}}
$$

where, $\bar{\zeta}=\left(\frac{\gamma(0)}{\theta} e^{-\left(\rho+\frac{\alpha}{\theta}\right) t_{q+1}}+\left(a_{0}+a_{1} e^{-t_{q+1}}\right)\right)$.

Proposition 2. Consider the case when $\|A\|=0$, then we obtain

$$
\left\|\dot{e}_{u}(t)\right\| \leq\|G\|\|B\|\left(t-t_{q}\right)
$$

which further satisfies

$\frac{\gamma(0)}{\theta} e^{-\left(\rho+\frac{\alpha}{\theta}\right) t}+\sigma\left(a_{0}+a_{1} e^{-b t}\right) \leq(\|G\|\|B\|)^{2}\left(t-t_{q}\right)^{2}$

Consider the next event is triggered at $t=t_{q+1}^{t h}$ time instant, inter-event time $t_{q+1}-t_{q}$ can be obtained from (47) as

$$
t_{q+1}-t_{q} \geq \ln \left[\frac{\bar{\zeta}}{\|G\|\|B\|}\right]^{\frac{1}{2}}
$$

where, $\bar{\zeta}=\left(\frac{\gamma(0)}{\theta} e^{-\left(\rho+\frac{\alpha}{\theta}\right) t_{q+1}}+\sigma\left(a_{0}+a_{1} e^{-b t_{q+1}}\right)\right)$.

\subsection{Effectiveness of design parameters}

For the given initial condition $\gamma(0)$, in this section we discuss how to chose the parameters for the triggering rule (7) so that we can improve the usage of resources.

4.1.1. Effect of $\theta$. (41) indicates that the minimum interevent time increases when the value of parameter $\theta$ decreases. However, for $\theta \rightarrow 0$, we get the maximum interevent time. For $\theta=\infty$, the lower bound of dynamic ETM is same as static ETM.

4.1.2. Effect of $\sigma$. For a fixed $\theta$, and constant scalars $\rho, \alpha$ and $a_{0}=0, \sigma$ measures how fast the system states converge to zero i.e. for larger $\sigma$, decaying rate is faster. 
4.1.3. Integral performance index. We consider an integral cost index to show the effect of parameters on system performance with respect to an integral index as follows.

$$
J=\int_{0}^{\infty} \frac{d u^{T}(t) u(t)}{d t} d t \leq \int_{0}^{\infty}\|G\|^{2} \frac{d\|x(t)\|^{2}}{d t} d t
$$

This kind of performance index is considered in [21] to show the rate of change in the control input. The above cost index shows that fluctuation in control input is less when the index value is less for the given interval. Substituting the bound on $\|x(t)\|$ from (27), we can notice that the upper bound of $J$ decreases when $\theta$ increases, i.e., fluctuation in control law is less for larger $\theta$ and thus, saves more resources.

\section{Simulation results}

This section presents the numerical validation of our proposed work. An example is considered from [1] for the linear continuous-time system as described by

$$
\dot{x}(t)=A x(t)+B u(t)
$$

where,

$$
A=\left[\begin{array}{cc}
0 & 1 \\
-2 & 3
\end{array}\right], B=\left[\begin{array}{l}
0 \\
1
\end{array}\right]
$$

Consider the initial condition of states are $x_{0}=\left[\begin{array}{ll}10 & 0\end{array}\right]^{T}$. $A$ is a state matrix and $B$ is an input matrix. Feedback gain $G=\left[\begin{array}{ll}1 & -4\end{array}\right]$. Initial condition of $\gamma(t)$ is fixed at $0 . \alpha, \rho$ are considered as $1 . a_{0}, a_{1}$ and $b$ are fixed at $0.003,0.01$ and 0.01 respectively. $\sigma$ is chosen 0.1 for Table II. In this simulation, several values of $\theta$ are considered to show its effect on inter-event time and control performance. To show the effectiveness of the proposed ETMs over the state-based ETM, we measure the system performance by integral error norm (such as integral square error (ITAE) [21].

$$
\text { ITAE }=\int_{t_{1}}^{t_{2}} t\left\|e_{u}(t)\right\|^{2} d t
$$

Fig. 2 shows the evolution of states, and control input for the proposed ETM (7) for $\theta=0.9$ respectively. Fig. 3 shows the number of events for different values of $\theta$. Note that the number of events for $\theta=\infty$ in DETM is the same as the number of events generated by the static ETM. It can also be noticed from Fig. 3 that inter-event time is significantly increased in the case of dynamic ETM when $\theta$ decreases. Table I shows that the proposed ETM (7) has less resource utilization than the state-based error. However, the mean inter-execution time for the proposed ETM (7) is larger than the state-based dynamic ETM. Table II shows the dynamic ETM (7) has larger mean inter-event time $\tau$ compared to the static ETM (6) for the given values of $a_{0}, a_{1}, \sigma$.

\section{Conclusion}

In this paper, an input-based dynamic event-triggered strategy has been presented. Due to the input-based error, system performance is improved by avoiding the redundant updates of control law, as shown in Table I. Also, due to
TABLE 1. CONTROL PERFORMANCE WITH DIFFERENT $\sigma$ FOR VARIOUS ETMs.

\begin{tabular}{|l|l|l|l|l|}
\hline Triggering mechanism & $\sigma$ & $\tau_{\text {mean }}$ & ITAE & J \\
\hline ETM (7) & 0.001 & $1.046 \mathrm{~s}$ & 0.341 & 0.838 \\
& 0.01 & $1.099 \mathrm{~s}$ & 0.738 & 0.854 \\
& 0.1 & $1.116 \mathrm{~s}$ & 0.935 & 0.857 \\
\hline $\begin{array}{l}\text { Proposed ETM with state- } \\
\text { based error }\end{array}$ & 0.001 & $0.45 \mathrm{~s}$ & 0.58 & 0.97 \\
& 0.01 & $0.452 \mathrm{~s}$ & 0.79 & 0.89 \\
& 0.1 & $0.463 \mathrm{~s}$ & 0.94 & 0.21 \\
\hline
\end{tabular}

TABLE 2. CONTROL PERFORMANCE WITH DIFFERENT $\theta$ FOR VARIOUS ETMS.

\begin{tabular}{|l|l|l|l|}
\hline Triggering mechanism & $\theta$ & $\tau_{\text {mean }}$ & $\mathbf{J}$ \\
\hline ETM (7) & 0.001 & $0.244 \mathrm{~s}$ & 0.838 \\
& 0.01 & $0.28 \mathrm{~s}$ & 0.8 \\
& 0.5 & $0.316 \mathrm{~s}$ & 0.786 \\
\hline Static ETM (6) & - & $0.01 \mathrm{~s}$ & 0.35 \\
\hline
\end{tabular}

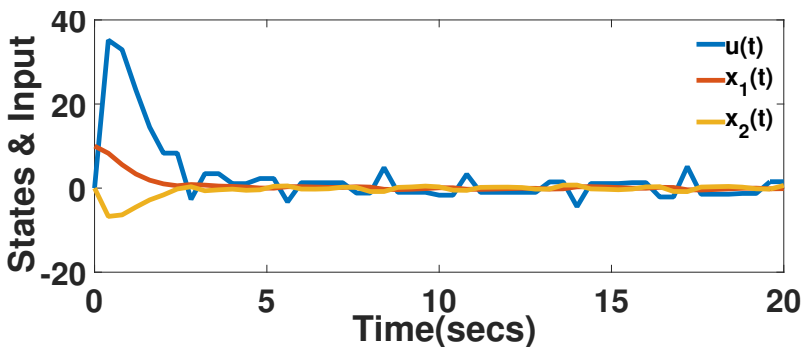

Figure 2. Illustration of system states \& control input for the triggering rule (7) with design parameters $\theta=0.9, \sigma=0.001, a_{0}=0.001, a_{1}=0.5$, and $b=0.1$

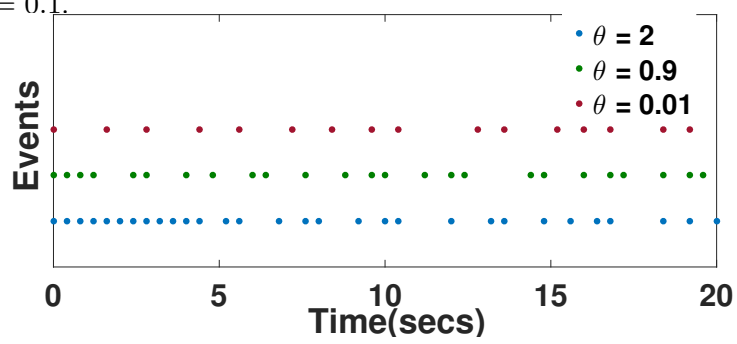

Figure 3. Events for different $\theta$ with design parameters $\sigma=0.001, a_{0}=$ $0.001, a_{1}=0.5$, and $b=0.1$.

introducing an auxiliary variable in the DETM, communication frequency has been reduced significantly than the static ETM, thus improving resource utilization. Inter-event time is positive for all values of $t>0$, which ensures that Zeno phenomena do not exist. System stability has been established using ISS criteria for the proposed triggering mechanism.

\section{References}

[1] P. Tabuada, "Event-triggered real-time scheduling of stabilizing control tasks," IEEE Transactions on Automatic Control, vol. 52, no. 9, pp. 1680-1685, 2007.

[2] M. Guinaldo, D. V. Dimarogonas, K. H. Johansson, J. Sánchez, and S. Dormido, "Distributed event-based control strategies for interconnected linear systems," IET Control Theory \& Applications, vol. 7, no. 6, pp. 877-886, 2013. 
[3] H. Yu and F. Hao, "Input-to-state stability of integral-based eventtriggered control for linear plants," Automatica, vol. 85, pp. 248-255, 2017.

[4] D. Borgers, V. Dolk, G. E. Dullerud, A. Teel, and W. Heemels, "Timeregularized and periodic event-triggered control for linear systems," in Control Subject to Computational and Communication Constraints. Springer, 2018, pp. 121-149.

[5] D. Liu and G.-H. Yang, "Dynamic event-triggered control for linear time-invariant systems with-gain performance," International Journal of Robust and Nonlinear Control, vol. 29, no. 2, pp. 507-518, 2019.

[6] B. A. Khashooei, D. J. Antunes, and W. Heemels, "Output-based event-triggered control with performance guarantees," IEEE Transactions on Automatic Control, vol. 62, no. 7, pp. 3646-3652, 2017.

[7] A. Girard, "Dynamic triggering mechanisms for event-triggered control," IEEE Transactions on Automatic Control, vol. 60, no. 7, pp. 1992-1997, 2014.

[8] W. Liu, Z. Wang, H. Dai, and M. Naz, "Dynamic output feedback control for fast sampling discrete-time singularly perturbed systems," IET Control Theory \& Applications, vol. 10, no. 15, pp. 1782-1788, 2016.

[9] X. Ge, Q.-L. Han, and Z. Wang, "A dynamic event-triggered transmission scheme for distributed set-membership estimation over wireless sensor networks," IEEE transactions on cybernetics, vol. 49, no. 1, pp. 171-183, 2017.

[10] Z. Gu, Z. Huan, D. Yue, and F. Yang, "Event-triggered dynamic output feedback control for networked control systems with probabilistic nonlinearities," Information Sciences, vol. 457, pp. 99-112, 2018.

[11] A. Molin and S. Hirche, "Adaptive event-triggered control over a shared network," in 2012 IEEE 51st IEEE Conference on Decision and Control (CDC). IEEE, 2012, pp. 6591-6596.

[12] W. He, B. Xu, Q.-L. Han, and F. Qian, "Adaptive consensus control of linear multiagent systems with dynamic event-triggered strategies," IEEE Transactions on Cybernetics, 2019.

[13] I. Karafyllis, N. Espitia, and M. Krstic, "Event-triggered gain scheduling of reaction-diffusion pdes," arXiv preprint arXiv:2005.13945, 2020.

[14] Y. Wu, X. Meng, L. Xie, R. Lu, H. Su, and Z.-G. Wu, "An inputbased triggering approach to leader-following problems," Automatica, vol. 75, pp. 221-228, 2017.

[15] Y. Xu, M. Fang, Z.-G. Wu, Y.-J. Pan, M. Chadli, and T. Huang, "Input-based event-triggering consensus of multiagent systems under denial-of-service attacks," IEEE Transactions on Systems, Man, and Cybernetics: Systems, 2018.

[16] W. Chenliang, L. Yun, H. Qinglei, and J. Huang, "Event-triggered adaptive control for attitude tracking of spacecraft," Chinese Journal of Aeronautics, vol. 32, no. 2, pp. 454-462, 2019.

[17] Y. Wang, Z. Jia, and Z. Zuo, "Dynamic event-triggered and selftriggered output feedback control of networked switched linear systems," Neurocomputing, vol. 314, pp. 39-47, 2018.

[18] W. Heemels, K. H. Johansson, and P. Tabuada, "Event-triggered and self-triggered control." 2015.

[19] E. D. Sontag, "Input to state stability: Basic concepts and results," in Nonlinear and optimal control theory. Springer, 2008, pp. 163-220.

[20] W. Wu, S. Reimann, D. Görges, and S. Liu, "Suboptimal eventtriggered control for time-delayed linear systems," IEEE Transactions on Automatic Control, vol. 60, no. 5, pp. 1386-1391, 2014.

[21] P. D. Domański, P. D. Domański, and Ditzinger, Control Performance Assessment: Theoretical Analyses and Industrial Practice. Springer, 2020. 